\title{
On Hom-Lie Pseudo-Superalgebras
}

\author{
Shengxiang Wang1,2, Tingting Tao ${ }^{*}$ \\ ${ }^{1}$ Department of Mathematics, Nanjing University, Nanjing, China \\ ${ }^{2}$ School of Mathematics and Finance, Chuzhou University, Chuzhou, China \\ Email: "14113697@qq.com
}

Received 19 March 2016; accepted 16 May 2016; published 19 May 2016

Copyright (C) 2016 by authors and Scientific Research Publishing Inc.

This work is licensed under the Creative Commons Attribution International License (CC BY). http://creativecommons.org/licenses/by/4.0/

(c) ()

\section{Abstract}

The aim of this article is to introduce the notion of Hom-Lie $\mathrm{H}$-pseudo-superalgebras for any Hopf algebra $\boldsymbol{H}$. This class of algebras is a natural generalization of the Hom-Lie pseudo-algebras as well as a special case of the Hom-Lie superalgebras. We present some construction theorems of HomLie $H$-pseudo-superalgebras, reformulate the equivalent definition of Hom-Lie $H$-pseudo-superalgebras, and consider the cohomology theory of Hom-Lie $\mathrm{H}$-pseudo-superalgebras with coefficients in arbitrary Hom-modules as a generalization of Kac's result.

\section{Keywords}

\section{Hom-Associative Pseudo-Superalgebra, Hom-Lie Pseudo-Superalgebra, Hom-Lie Conformal Superalgebra, Hom-Annihilation Superalgebra, Cohomology}

\section{Introduction}

The notion of conformal algebras [1]-[5] was introduced by Kac as a formal language describing the singular part of the operator product expansion in two-dimensional conformal field theory, and it came to be useful for investigation of vertex algebras (see [6]-[8]). The concept of vertex algebras was derived from mathematical physics; it was first mathematically defined and considered by Borcherds in [9] to obtain his solution of the Moonshine conjecture in the theory of finite simple groups.

As a generalization of conformal algebras, Bakalov, D'Andrea and Kac [10] developed a theory of "multidimensional" lie conformal algebras, called Lie $H$-pseudo-algebras for any Hopf algebra $H$. Classification problems, cohomology theory and representation theory have been considered in [10]-[12]. In [13], Boyallian and Liberati studied pseudo-algebras from the point of view of pseudo-dual of classical Lie coalgebra structures by defining the notions of Lie $H$-coalgebras and Lie pseudo-bialgebras.

In [14], Sun generalized the pseudo-algebra structures to the Hom-pseudo-algebras of associative and Lie type,

\footnotetext{
*Corresponding author.
} 
and showed some examples of the new structures and construction theorems. Hom-algebras were firstly studied by Hartwig, Larsson and Silvestrov in [15], where they introduced the structure of Hom-Lie algebras in the context of the deformations of Witt and Virasoro algebras. Later, Larsson and Silvestrov extended the notion of Hom-Lie algebras to quasi-Hom Lie algebras and quasi-Lie algebras (see [16]). Recently, Yau laid the foundation of a homology theory for Hom-Lie algebras and constructed the enveloping algebras of Hom-Lie and Hom-Leibniz algebras in [17]-[19]. Many more properties and structures of Hom-Lie algebras have been developed (see [20]-[23] and references cited therein).

In [24], Hom-algebras and Hom-coalgebras were introduced by Makhlouf and Silvestrov as a generalization of ordinary algebras and coalgebras in the following sense: the associativity of the multiplication was replaced by the Hom-associativity and similar for Hom-coassociativity. They also defined the structures of Hom-bialgebras and Hom-Hopf algebras, and described some of their properties extending properties of ordinary bialgebras and Hopf algebras in [25] and [26]. Different to Makhlouf and Silvestrov's work, Caenepeel and Goyvaerts studied the Hom-Hopf algebras from a categorical view point in [27], and called them monoidal Hom-bialgebras and monoidal Hom-Hopf algebras respectively (for more details about monoidal Hom-Hopf algebras, see references [28]-[32] and references cited therein).

In [33], Ammar and Makhlouf introduced the notion of Hom-Lie superalgebras and provided a construction theorem from which one can derive a one parameter family of Hom-Lie superalgebras deforming the orthosymplectic Lie superalgebras. The notion of Hom-Lie superalgebras is a natural and meaningful generalization of Lie superalgebras which were introduced by Kac in [3]. Motivated by [4] [10], in which Kac formulated the notion of conformal superalgebras and considered the classification theorem and representation theory of conformal superalgebras. We think whether we can extend the notions of Hom-Lie pseudo-algebras and conformal superalgebras to Hom-Lie pseudo-superalgebras.

Cohomology is an important tool in mathematics. Its range of applications contains algebra and topology as well as the theory of smooth manifolds or of holomorphic functions. The cohomology theory of Lie algebras was developed by Chevalley, Eilenberg and Cartan. Scheunert and Zhang introduced and investigated the cohomology groups of Lie superalgebras in [34]. Naturally, we think whether we can extend the notion of cohomology groups to Hom-Lie $H$-pseudo-superalgebras. This becomes our second motivation of the paper.

To give a positive answer to the questions above, we organize this paper as follows. In Section 2, we recall some basic definitions about Lie pseudo-algebras. In Section 3, we define Hom-Lie pseudo-superalgebras and introduce two construction theorems of Hom-Lie pseudo-superalgebras (see Proposition 3.12 and Theorem 3.13). In Section 4, we mainly discuss the annihilation superalgebras of Hom-pseudo-superalgebras (see Proposition 4.5). In Section 5, we determine some equivalent definitions of Hom-pseudo-superalgebras. In Section 6, we discuss the cohomology of Hom-Lie $H$-pseudo-superalgebras (see Theorem 6.1).

\section{Preliminaries}

In this section we recall some basic definitions and results related to our paper. Throughout the paper, all algebraic systems are supposed to be over a field $k$ of characteristic $0, H$ always denotes a Hopf algebra with an antipode $S$. We summarize in the following the ungraded definitions of Hom-associative and Hom-Lie $H$-pseudoalgebras (see [14]). The reader is referred to Sweedler [35] about Hopf algebras, the Sweedler-type notation for the comultiplication is denoted by: $\Delta(x)=x_{(1)} \otimes x_{(2)}$.

Recall that a pseudotensor category $M^{*}(H)$ is a category whose objects are the same objects as in the category ${ }_{H} M$ of left $H$-modules, but with a non-trivial pseudotensor structure, see [10].

A Hom-associative $H$-pseudo-algebra [14] is a triple $(A, \mu=*, \alpha)$ consisting of a linear space $A$ in $M^{*}(H)$, an operation $\mu \in \operatorname{Hom}_{H^{\otimes 2}}\left(A \otimes A, H^{\otimes 2} \otimes_{H} A\right)$ and a homomorphism $\alpha \in \operatorname{Hom}_{H}(A, A)$ satisfying

$$
\alpha(a) *(b * c)=(a * b) * \alpha(c), a, b, c \in A \text {. }
$$

A Hom-Lie $H$-pseudo-algebra [14] is a triple $(L, \mu=[*], \alpha)$ consisting of a linear space $L$ in $M^{*}(H)$, an operation $\mu \in \operatorname{Hom}_{H^{\otimes 2}}\left(L \otimes L, H^{\otimes 2} \otimes_{H} L\right)$ and a homomorphism $\alpha \in \operatorname{Hom}_{H}(L, L)$ satisfying the following axioms $(a, b, c \in L)$ :

1) Skew-commutativity:

$$
[b * a]=-\left(\sigma \otimes_{H} i d\right)[a * b]
$$

2) Hom-Jacobi identity: 


$$
[\alpha(a) *[b * c]]-\left((\sigma \otimes i d) \otimes_{H} i d\right)[\alpha(b) *[a * c]]=[[a * b] * \alpha(c)] .
$$

An elementary but important property of Hom-Lie $H$-pseudo-algebra is that each Hom-associative $H$-pseudoalgebra gives rise to a Hom-Lie $H$-pseudo-algebra via the commutator bracket.

A Hom-Lie $H$-conformal algebra ([14]) is a triple $(L,[],, \alpha)$ consisting of a linear space $L$ in ${ }_{H} M$, an oper-

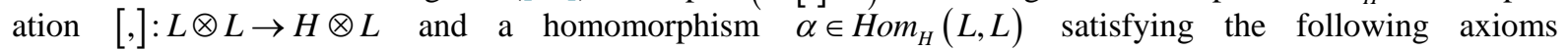
( $a, b, c \in L$ and $h \in H$ ):

1) $H$-sesqui-linearity:

$$
[h a, b]=(h \otimes 1)[a, b],[a, h b]=\left(1 \otimes h_{(2)}\right)[a, b]\left(S\left(h_{(1)}\right) \otimes 1\right) .
$$

2) Skew-commutativity:

$$
[b, a]=-\sum_{i} S\left(h_{i_{(1)}}\right) \otimes h_{i_{(2)}} c_{i} \text {, if }[a, b]=\sum_{i} h_{i} \otimes c_{i}
$$

3) Hom-Jacobi identity:

$$
[\alpha(a),[b, c]]-(\sigma \otimes i d)[\alpha(b),[a, c]]=\left(F^{-1} \otimes i d\right)[[a, b], \alpha(c)] .
$$

Recall from Sun [14] we know that one can reformulate the definition of a Hom-Lie $H$-pseudo-algebra via a Hom-Lie $H$-conformal algebra.

\section{Hom-Pseudo Superalgebras of Associative and Lie Types}

In this section we will introduce the concept and construction theorems of Hom- $H$-pseudo-superalgebras of associative and Lie types, and show some examples of Hom-Lie $H$-pseudo-superalgebras that are neither Hom-Lie superalgebras nor Hom-Lie pseudo-algebras.

Definition 3.1. A Hom-associative H-pseudo-superalgebra is a triple $(A, \mu=*, \alpha)$ consisting of a superspace $A$ in $M^{*}(H)$, an even operation $\mu \in H_{H^{\circ 2}}\left(A \otimes A, H^{\otimes 2} \otimes_{H} A\right)$ and an even homomorphism $\alpha \in \operatorname{Hom}_{H}(A, A)$ satisfying

$$
\alpha(a) *(b * c)=(a * b) * \alpha(c)
$$

in $H^{\otimes 3} \otimes_{H} A$ for all homogeneous elements $a, b, c \in A$.

Example 3.2. For a one dimensional Hopf algebra $H=k$, a Hom-associative $H$-pseudo-superalgebra is just a Hom-associative superalgebra over $k$. If $\alpha=i d$, then a Hom-associative $H$-pseudo-superalgebra is an associative H-pseudo-superalgebra.

A Hom-associative $H$-pseudo-superalgebra $(A, \mu, \alpha)$ is called multiplicative if $\left(i d_{H^{\otimes 2}} \otimes_{H} \alpha\right) \mu=\mu(\alpha \otimes \alpha)$. For example, if $\alpha=i d_{A}$, then the Hom-associative $H$-pseudo-superalgebra $(A, \mu, \alpha)$ is multiplicative.

Let $\left(A, \mu_{A}, \alpha_{A}\right)$ and $\left(B, \mu_{B}, \alpha_{B}\right)$ be two (multiplicative) Hom-associative $H$-pseudo-superalgebras, an even homomorphism $f: A \rightarrow B$ is said to be a morphism of Hom-associative $H$-pseudo-superalgebras if

$$
\left(i d_{H^{\otimes 2}} \otimes_{H} f\right) \mu_{A}=\mu_{B}(f \otimes f), f \alpha_{A}=\alpha_{B} f .
$$

Definition 3.3. Let $(A, *, \alpha)$ be a Hom-associative $H$-pseudo-superalgebra and $M$ be a superapace in ${ }_{H} M$. A Hom- $A$-module is a triple $\left(M, \rho_{M}, \alpha_{M}\right)$, where $\rho_{M}$ is an even morphism in $\operatorname{Hom}_{H^{\otimes 2}}\left(A \otimes M, H^{\otimes 2} \otimes_{H} M\right)$, $\alpha_{M}$ is an even morphism in $\operatorname{Hom}_{H}(M, M)$ and satisfies the following properties $(a, b \in A, m \in M)$ :

$$
\begin{gathered}
\alpha_{A}(a) *(b * m)=(a * b) * \alpha_{M}(m), \\
\left(i d_{H^{\otimes 2}} \otimes_{H} \alpha_{M}\right)(a * m)=\alpha_{A}(a) * \alpha_{M}(m),
\end{gathered}
$$

where $a * m=\rho_{M}(a \otimes m)$.

Example 3.4. Let $(A, \alpha)$ be a finite dimensional Hom-associative superalgebra, $H$ be a Hopf algebra. Then $(H \otimes A, *, i d \otimes \alpha)$ is a Hom-associative $H$-pseudo-superalgebra with pseudoproduct $*$ given by

$$
(f \otimes a) *(g \otimes b)=f \otimes g \otimes\left(1 \otimes_{H} a b\right)
$$

for all $f, g \in H$ and homogeneous elements $a, b \in A$. 
Definition 3.5. A Hom-Lie $H$-pseudo-superalgebra is a triple $(L, \mu=[*], \alpha)$ consisting of a superspace $L$ in $M^{*}(H)$, an even operation $\mu \in \operatorname{Hom}_{H^{\otimes 2}}\left(L \otimes L, H^{\otimes 2} \otimes_{H} L\right)$ and an even homomorphism $\alpha \in \operatorname{Hom}_{H}(L, L)$ satisfying the following axioms:

1) Skew-commutativity:

$$
[b * a]=-(-1)^{|a| b \mid}\left(\sigma \otimes_{H} i d\right)[a * b]
$$

2) Hom-Jacobi identity:

$$
[\alpha(a) *[b * c]]-(-1)^{|a| b \mid}\left((\sigma \otimes i d) \otimes_{H} i d\right)[\alpha(b) *[a * c]]=[[a * b] * \alpha(c)],
$$

where $a, b, c$ are homogeneous elements in $L$.

Here and further, $|a|$ is the parity of $a$.

Example 3.6. For a one dimensional Hopf algebra $H=k$, a Hom-Lie $H$-pseudo-superalgebra is just a Hom-Lie superalgebra over $k$. If $\alpha=i d$, then a Hom-Lie $H$-pseudo-superalgebra is a Lie $H$-pseudo-superalgebra.

Example 3.7. Let $H$ be a Hopf algebra and $A=A_{0}+A_{1}$ a 2-dimensional linear superspace, where $A_{0}$ is generated by $x$ and $A_{1}$ is generated by $y$. Then $(L=H\{x, y\},[*], \alpha)$ is a Hom-Lie $H$-pseudo-superalgebra, where $H\{x, y\}$ is a free pseudo-algebra of rank 2 with pseudoproduct given by $[x * x]=[y * y]=[x * y]=0$ in $H^{\otimes 2} \otimes_{H} L, \alpha$ is any even homomorphism in $\operatorname{Hom}_{H}(L, L)$.

Example 3.8. Let $(L,[],, \alpha)$ be a finite dimensional Hom-Lie superalgebra, $H$ be a Hopf algebra. Then $(H \otimes L,[*], i d \otimes \alpha)$ is a Hom-Lie $H$-pseudo-superalgebra with pseudoproduct [*] given by

$$
[(h \otimes x) *(l \otimes y)]=h \otimes l \otimes_{H}(1 \otimes[x, y])
$$

for all $h, l \in H$ and homogeneous elements $x, y \in L$.

Example 3.9. Let $H$ be a Hopf algebra and $A=A_{0}+A_{1}$ a 3-dimensional linear superspace, where $A_{0}$ is generated by $x, y$ and $A_{1}$ is generated by $z$. Then $(L=H\{x, y, z\},[*], \alpha)$ is a Hom-Lie H-pseudo-superalgebra defined by any even homomorphism $\alpha$ and operation

$$
\begin{gathered}
{[x * y]=1 \otimes h \otimes x,[y * x]=-h \otimes 1 \otimes x, h \in H,} \\
{[x * x]=[y * y]=[z * z]=[x * z]=[z * x]=[y * z]=[z * y]=0 .}
\end{gathered}
$$

In particular, if $H=k$, then the Hom-Lie $H$-pseudo-superalgebra $(L=H\{x, y, z\},[*], \alpha)$ is noting but the affine Hom-Lie superalgebra in [33].

A Hom-Lie $H$-pseudo-superalgebra $(L,[*], \alpha)$ is called multiplicative if $\left(i_{H^{\otimes 2}} \otimes_{H} \alpha\right)[*]=[*](\alpha \otimes \alpha)$. For example, if $\alpha=i d_{A}$, then the Hom-Lie $H$-pseudo-superalgebra $(A, \mu, \alpha)$ is multiplicative.

Let $\left(A,[*]_{A}, \alpha_{A}\right)$ and $\left(B,[*]_{B}, \alpha_{B}\right)$ be two (multiplicative) Hom-Lie $H$-pseudo-superalgebras. An even homomorphism $f: A \rightarrow B$ is said to be a morphism of Hom-Lie $H$-pseudo-superalgebras if

$$
\left(i d_{H^{\otimes 2}} \otimes_{H} f\right)[*]_{A}=[*]_{B}(f \otimes f), f \alpha_{A}=\alpha_{B} f .
$$

Definition 3.10. Let $\left(L,[*], \alpha_{L}\right)$ be a Hom-Lie $H$-pseudo-superalgebra and $M$ a superspace in ${ }_{H} M$. A Hom- $L$-module is a triple $\left(M, \rho_{M}, \alpha_{M}\right)$, where $\rho_{M}$ is an even morphism in $H_{o m} H_{H^{\otimes 2}}\left(L \otimes M, H^{\otimes 2} \otimes_{H} M\right)$, $\alpha_{M}$ is an even morphism in $\operatorname{Hom}_{H}(M, M)$ and satisfies the following axioms:

$$
\alpha_{L}(a) *(b * m)=[a * b] * \alpha_{M}(m),\left(i d_{H^{\otimes 2}} \otimes_{H} \alpha_{M}\right)(a * m)=\alpha_{L}(a) * \alpha_{M}(m),
$$

where $a * m=\rho_{M}(a \otimes m), a, b$ and $m$ are homogeneous elements in $L$ and $M$ respectively.

In the following, we will show that the supercommutator bracket defined using the multiplication in a Homassociative $H$-pseudo-superalgebra leads naturally to a Hom-Lie $H$-pseudo-superalgebra.

Lemma 3.11. Let $(A, *, \alpha)$ be a Hom-associative $H$-pseudo-superalgebra. Then

1) $\left((\sigma \otimes i d) \otimes_{H} i d\right)(\alpha(a) *(b * c))=((\sigma \otimes i d)(a * b)) * \alpha(c)$.

2) $\left((i d \otimes \sigma) \otimes_{H} i d\right)((a * b) * \alpha(c))=\alpha(a) *((\sigma \otimes i d)(b * c))$.

3) $\left((\sigma \otimes i d) \otimes_{H} i d\right)\left((i d \otimes \sigma) \otimes_{H} i d\right)(((\sigma \otimes i d)(a * b)) * \alpha(c))$

$$
=\left((i d \otimes \sigma) \otimes_{H} i d\right)\left((\sigma \otimes i d) \otimes_{H} i d\right)(\alpha(a) *((\sigma \otimes i d)(b * c))) .
$$


Proof. We only prove (3), and similarly for (1), (2). For any homogeneous elements $a, b, c \in A$, let

$$
\begin{gathered}
a * b=\sum_{i} f_{i} \otimes g_{i} \otimes e_{i}, e_{i} * \alpha(c)=\sum_{i, j} f_{i, j} \otimes g_{i, j} \otimes e_{i, j}, \\
b * c=\sum_{i} h_{i} \otimes l_{i} \otimes d_{i}, \alpha(a) * d_{i}=\sum_{i, j} h_{i, j} \otimes l_{i, j} \otimes d_{i, j} .
\end{gathered}
$$

On one hand we have

$$
\begin{aligned}
& \left((\sigma \otimes i d) \otimes_{H} i d\right)\left((i d \otimes \sigma) \otimes_{H} i d\right)(((\sigma \otimes i d)(a * b)) * \alpha(c)) \\
& =\sum_{i, j}\left((\sigma \otimes i d) \otimes_{H} i d\right)\left((i d \otimes \sigma) \otimes_{H} i d\right)\left(\left(g_{i} f_{i j(1)} \otimes f_{i} f_{i j(2)} \otimes g_{i j}\right) \otimes_{H} e_{i j}\right) \\
& =\sum_{i, j}\left((\sigma \otimes i d) \otimes_{H} i d\right)\left(\left(g_{i} f_{i j(1)} \otimes g_{i j} \otimes f_{i} f_{i j(2)}\right) \otimes_{H} e_{i j}\right) \\
& =\sum_{i, j}\left(g_{i j} \otimes g_{i} f_{i j(1)} \otimes f_{i} f_{i j_{(2)}}\right) \otimes_{H} e_{i j}=\sum_{i, j}\left(g_{i j} \otimes g_{i} f_{i j(2)} \otimes f_{i} f_{i j(1)}\right) \otimes_{H} e_{i j},
\end{aligned}
$$

since $H$ is cocommutative. Similarly, we have

$$
\begin{aligned}
& \left((i d \otimes \sigma) \otimes_{H} i d\right)\left((\sigma \otimes i d) \otimes_{H} i d\right)(\alpha(a) *((\sigma \otimes i d)(b * c))) \\
& =\left((i d \otimes \sigma) \otimes_{H} i d\right)\left((\sigma \otimes i d) \otimes_{H} i d\right)\left(\left(h_{i j} \otimes l_{i} l_{i j(1)} \otimes h_{i} l_{i j_{(2)}}\right) \otimes_{H} d_{i j}\right) \\
& =\sum_{i, j}\left((i d \otimes \sigma) \otimes_{H} i d\right)\left(\left(l_{i} l_{i j(1)} \otimes h_{i j} \otimes h_{i} l_{i j(2)}\right) \otimes_{H} d_{i j}\right) \\
& =\sum_{i, j}\left(l_{i} l_{i j(1)} \otimes h_{i} l_{i j(2)} \otimes h_{i j}\right) \otimes_{H} d_{i j}=\sum_{i, j}\left(l_{i} l_{i j(2)} \otimes h_{i} l_{i j_{(1)}} \otimes h_{i j}\right) \otimes_{H} d_{i j},
\end{aligned}
$$

as required. So (3) holds since $A$ is Hom-associative.

Proposition 3.12. Given any Hom-associative $H$-pseudo-superalgebra $(A, *, \alpha)$, one can define the bracket pseudoproduct on homogeneous elements by

$$
[a * b]=a * b-(-1)^{|a| b \mid}\left(\sigma \otimes_{H} i d\right)(b * a)
$$

and then extending by linearity to all elements. Then $(A,[*], \alpha)$ is a Hom-Lie $H$-pseudo-superalgebra.

Proof. We shall show that the condition (3.9) leads A to be a Hom-Lie $H$-pseudo-superalgebra, in the sense of Definition 3.5. For this purpose, we first claim that the bracket pseudoproduct is both $H$-bilinear and skewcommutative, but these are easy to check. It remains to verify that the conditions (2) of Definition 3.5 are satisfied by the condition (3.9). Now we have the following calculations:

$$
\begin{aligned}
& {[\alpha(a) *[b * c]]=\left[\alpha(a) *\left(b * c-(-1)^{|b| c \mid}\left(\sigma \otimes_{H} i d\right)(c * b)\right)\right]} \\
& =[\alpha(a) *(b * c)]-(-1)^{|b||c|}\left[\alpha(a) *\left(\left(\sigma \otimes_{H} i d\right)(c * b)\right)\right] \\
& =\alpha(a) *(b * c)-(-1)^{b|| c \mid} \alpha(a) *\left(\left(\sigma \otimes_{H} i d\right)(c * b)\right) \\
& -(-1)^{\mid a(|b++c|)}\left((\sigma \otimes i d) \otimes_{H} i d\right)\left((i d \otimes \sigma) \otimes_{H} i d\right)((b * c) * \alpha(a)) \\
& +(-1)^{|b| c|c| a \mid(|b|+|c|)}\left((\sigma \otimes i d) \otimes_{H} \text { id }\right)\left((i d \otimes \sigma) \otimes_{H} \text { id }\right)(((\sigma \otimes i d)(c * b)) * \alpha(a)) \\
& =\alpha(a) *(b * c)-(-1)^{|b| c \mid}\left((i d \otimes \sigma) \otimes_{H} i d\right)((a * c) * \alpha(b)) \\
& -(-1)^{\mid a(|b|+c \mid)}\left((\sigma \otimes i d) \otimes_{H} i d\right)(\alpha(b) *(\sigma \otimes i d)(c * a)) \\
& +(-1)^{b|c| c+|a|(|b+| c \mid)}\left((\sigma \otimes i d) \otimes_{H} \text { id }\right)\left((i d \otimes \sigma) \otimes_{H} \text { id }\right)(((\sigma \otimes i d)(c * b)) * \alpha(a)) .
\end{aligned}
$$

Immediately, we can obtain $[\alpha(b) *[a * c]]$, then 


$$
\begin{aligned}
& (-1)^{|a| b \mid}\left((\sigma \otimes i d) \otimes_{H} i d\right)[\alpha(b) *[a * c]] \\
& =(-1)^{|a| b \mid}\left((\sigma \otimes i d) \otimes_{H} i d\right)(\alpha(b) *(a * c)) \\
& \quad-(-1)^{|b| c \mid}\left((i d \otimes \sigma) \otimes_{H} i d\right)((a * c) * \alpha(b)) \\
& \quad-(-1)^{|a|(|b|+|c|)}\left((\sigma \otimes i d) \otimes_{H} \text { id }\right)(\alpha(b) *(\sigma \otimes i d)(c * a)) \\
& \quad+(-1)^{|c|(|a|+b \mid)}\left((i d \otimes \sigma) \otimes_{H} \text { id }\right)((\sigma \otimes i d)(c * a) * \alpha(b)) .
\end{aligned}
$$

It follows from Lemma 3.12 that

$$
\begin{aligned}
& {[\alpha(a) *[b * c]]-(-1)^{|a| b \mid}\left((\sigma \otimes i d) \otimes_{H} \text { id }\right)[\alpha(b) *[a * c]]} \\
& =(a * b) * \alpha(c)-(-1)^{|a| b \mid}\left((\sigma \otimes i d) \otimes_{H} \text { id }\right)((b * a) * \alpha(c)) \\
& \quad-(-1)^{|c||a|+|b|)}\left((i d \otimes \sigma) \otimes_{H} i d\right)((\sigma \otimes i d)(c * a) * \alpha(b)) \\
& \quad+(-1)^{|a| b|+| c|| a++b|| c \mid}\left((\sigma \otimes i d) \otimes_{H} \text { id }\right)\left((i d \otimes \sigma) \otimes_{H} i d\right)((\sigma \otimes i d)(c * b) * \alpha(a)) .
\end{aligned}
$$

Furthermore, we have

$$
\begin{aligned}
& {[[a * b] * \alpha(c)]=[(a * b) * \alpha(c)]-(-1)^{|a| b \mid}[(\sigma \otimes i d)(a * b) * \alpha(c)]} \\
& =(a * b) * \alpha(c)-(-1)^{|a| b \mid}(\sigma \otimes i d)(b * a) * \alpha(c) \\
& -(-1)^{|c|(|a|+b \mid)}\left((\text { id } \otimes \sigma) \otimes_{H} \text { id }\right)\left((\sigma \otimes i d) \otimes_{H} \text { id }\right)((c * a) * \alpha(b)) \\
& +(-1)^{|a| b|+| c|a|+b|| c \mid}\left((i d \otimes \sigma) \otimes_{H} \text { id }\right)\left((\sigma \otimes i d) \otimes_{H} \text { id }\right)(\alpha(c) *(\sigma \otimes i d)(b * a)) \\
& =(a * b) * \alpha(c)-(-1)^{|a||b|}\left((\sigma \otimes i d) \otimes_{H} i d\right)((b * a) * \alpha(c)) \\
& -(-1)^{|c|(|a|+|b|)}\left((i d \otimes \sigma) \otimes_{H} i d\right)((\sigma \otimes i d)(c * a) * \alpha(b)) \\
& +(-1)^{|a| b|+| c|| a|+b||c|}\left((\sigma \otimes i d) \otimes_{H} i d\right)\left((i d \otimes \sigma) \otimes_{H} i d\right)((\sigma \otimes i d)(c * b) * \alpha(a)) .
\end{aligned}
$$

Together with the above results, we finally obtain

$$
\begin{aligned}
& {[\alpha(a) *[b * c]]-(-1)^{|a||b|}\left((\sigma \otimes i d) \otimes_{H} i d\right)[\alpha(b) *[a * c]]} \\
& =[[a * b] * \alpha(c)] .
\end{aligned}
$$

The proof is completed.

Next we will construct Hom-Lie $H$-pseudo-superalgebras from Lie $H$-pseudo-superalgebras and even HomLie superalgebra endomorphisms, generalizing the results for Hom-Lie $H$-pseudo-algebras in [14] and Hom-Lie superalgebras in [33].

Theorem 3.13. Let $(L,[*])$ be a Lie $H$-pseudo-superalgebra and $\alpha$ an even endomorphisms of $L$. Defining $[*]_{\alpha} \in \operatorname{Hom}_{H^{\otimes 2}}\left(L^{\otimes 2}, H^{\otimes 2} \otimes_{H} L\right)$ by $[x * y]_{\alpha}=[\alpha(x) * \alpha(y)]$ for all homogeneous elements $x, y$ in $L$, then $\left(L,[*]_{\alpha}, \alpha\right)$ is a Hom-Lie $H$-pseudo-superalgebra.

Moreover, suppose that $\left(L^{\prime},[*]^{\prime}\right)$ is another Lie $H$-pseudo-superalgebra and $\alpha^{\prime}$ is an even endomorphisms of $L^{\prime}$. If $f: L \rightarrow L^{\prime}$ is a morphism of Lie $H$-pseudo-superalgebras that satisfies $f \alpha=\alpha^{\prime} f$, then

$$
f:\left(L,[*]_{\alpha}, \alpha\right) \rightarrow\left(L,[*]_{\alpha^{\prime}}^{\prime}, \alpha^{\prime}\right)
$$

is a morphism of Hom-Lie $H$-pseudo-superalgebras.

Proof. We shall show that $\left(L,[*]_{\alpha}, \alpha\right)$ satisfies the skew-commutativity and the Hom-Jacobi identity. For any homogeneous elements $x, y, z$ in $L$, 


$$
\begin{aligned}
{[x * y]_{\alpha} } & =\left(i d_{H^{\otimes 2}} \otimes_{H} \alpha\right)[x * y] \\
& =-(-1)^{|x| y \mid}\left(i d_{H^{\otimes 2}} \otimes_{H} \alpha\right)\left(\sigma \otimes_{H} i d\right)[y * x] \\
& =-(-1)^{|x| y \mid}\left(\sigma \otimes_{H} i d\right)\left(i d_{H^{\otimes 2}} \otimes_{H} \alpha\right)[y * x] \\
& =-(-1)^{|x| y \mid}\left(\sigma \otimes_{H} i d\right)[y * x]_{\alpha} .
\end{aligned}
$$

Since $\alpha$ is an endomorphism of $L$,

Therefore we have

$$
\begin{aligned}
{\left[\alpha(x) *[y * z]_{\alpha}\right]_{\alpha} } & =\left[\alpha(x) *\left(i d_{H^{\otimes 2}} \otimes_{H} \alpha\right)[y * z]\right]_{\alpha} \\
& =\left(i d_{H^{\otimes 3}} \otimes_{H} \alpha^{2}\right)[x *[y * z]] .
\end{aligned}
$$

$$
\begin{aligned}
& {\left[\alpha(x) *[y * z]_{\alpha}\right]_{\alpha}-\left[[x * y]_{\alpha} * \alpha(z)\right]_{\alpha}-(-1)^{|x| y \mid}\left((\sigma \otimes i d) \otimes_{H} i d\right)\left[\alpha(y) *[x * z]_{\alpha}\right]_{\alpha}} \\
& =\left(i d_{H^{\otimes 3}} \otimes_{H} \alpha^{2}\right)\left([x *[y * z]]-[[x * y] * z]-(-1)^{|x| y \mid}\left((\sigma \otimes i d) \otimes_{H} i d\right)[y *[x * z]]\right) \\
& =0
\end{aligned}
$$

as needed. To show that $f$ is a morphism of Hom-Lie $H$-pseudo-superalgebras, we do the calculations:

$$
\begin{aligned}
& \left(i d_{H^{\otimes 2}} \otimes f\right)\left([x * y]_{\alpha}\right)=\left(i d_{H^{\otimes 2}} \otimes f\right)\left(\left(i d_{H^{\otimes 2}} \otimes_{H} \alpha\right)[x * y]\right) \\
& =\left(\left(i d_{H^{\otimes 2}} \otimes_{H} f \alpha\right)[x * y]\right)=\left(\left(i d_{H^{\otimes 2}} \otimes_{H} \alpha^{\prime} f\right)[x * y]\right) \\
& =\left(i d_{H^{\otimes 2}} \otimes \alpha^{\prime}\right)\left(\left(i d_{H^{\otimes 2}} \otimes_{H} f\right)[x * y]\right) \\
& =\left(i d_{H^{\otimes 2}} \otimes \alpha^{\prime}\right)[f(x) * f(y)]^{\prime} \\
& =[f(x) * f(y)]_{\alpha^{\prime}} .
\end{aligned}
$$

The proof is completed.

To provides another way to construct Hom-Lie $H$-pseudo-superalgebras and Hom-associative $H$-pseudosuperalgebras, we first recall the definition of current $H$-pseudo-algebras in [10].

Let $H^{\prime}$ be a Hopf subalgebra of $H$ and $A$ an $H^{\prime}$-pseudo-algebra. Then define the current $H$-pseudo-algebra $\operatorname{Cur} A=H \otimes_{H^{\prime}} A$ by extending the pseudoproduct $a * b$ of $A$ using the $H$-bilinearity. Explicitly, for any $a, b \in A$, define

$$
\begin{aligned}
\left(f \otimes_{H^{\prime}} a\right) *\left(g \otimes_{H^{\prime}} b\right) & =\left(f \otimes g \otimes_{H} 1\right)(a * b) \\
& =\sum_{i}\left(f f_{i} \otimes g g_{i}\right) \otimes_{H}\left(1 \otimes_{H^{\prime}} e_{i}\right)
\end{aligned}
$$

if $a * b=f_{i} \otimes g_{i} \otimes_{H^{\prime}} e_{i}$. Then $\operatorname{CurA}=H \otimes_{H^{\prime}} A$ is an $H$-pseudo-algebra which is Lie or associative when $A$ is so.

Proposition 3.14. Let $H^{\prime}$ be a Hopf subalgebra of $H$ and $(L,[*], \alpha)$ a Hom-Lie $H^{\prime}$-pseudo-superalgebra. Then (CurL, $\gamma, \beta=i d_{H} \otimes_{H^{\prime}} \alpha$ ) is a Hom-Lie $H$-pseudo-superalgebra, where $\gamma$ is the multiplication of CurL. Moreover, there is a similar result in the case of Hom-associative $H^{\prime}$-pseudo-superalgebras as well.

Proof. We only prove the case of Hom-Lie $H^{\prime}$-pseudo-superalgebras, the Hom-associative case is similar. We denote

$$
\gamma\left(\left(f \otimes_{H^{\prime}} a\right) \otimes\left(g \otimes_{H^{\prime}} b\right)\right)=\left[\left(f \otimes_{H^{\prime}} a\right) *\left(g \otimes_{H^{\prime}} b\right)\right] .
$$

It is obviously that the skew-commutativity holds since $(L,[*], \alpha)$ is a Hom-Lie $H^{\prime}$-pseudo-superalgebra. So it is sufficient to verify the Hom-Jacobi identity. For any $f \otimes_{H^{\prime}} a, g \otimes_{H^{\prime}} b, l \otimes_{H^{\prime}} c \in C u r L$, suppose

$$
b * c=\sum_{i} g_{i} \otimes l_{i} \otimes_{H^{\prime}} d_{i}, \alpha(a) * d_{i}=\sum_{j} g_{i j} \otimes l_{i j} \otimes_{H^{\prime}} d_{i j},
$$




$$
\begin{gathered}
a * c=\sum_{i} m_{i} \otimes n_{i} \otimes_{H^{\prime}} e_{i}, \alpha(b) * e_{i}=\sum_{j} m_{i j} \otimes n_{i j} \otimes_{H^{\prime}} e_{i j}, \\
a * b=\sum_{i} s_{i} \otimes t_{i} \otimes_{H^{\prime}} u_{i}, u_{i} * \alpha(c)=\sum_{j} s_{i j} \otimes t_{i j} \otimes_{H^{\prime}} u_{i j} .
\end{gathered}
$$

Since $(L,[*], \alpha)$ is a Hom-Lie $H^{\prime}$-pseudo-superalgebra, we have

$$
[\alpha(a) *[b * c]]-(-1)^{|a| b \mid}\left((\sigma \otimes i d) \otimes_{H^{\prime}} \text { id }\right)[\alpha(b) *[a * c]]=[[a * b] * \alpha(c)],
$$

that is,

$$
\begin{aligned}
& \sum_{i, j} g_{i j} \otimes g_{i} l_{i j_{(1)}} \otimes g_{i} l_{i j(2)} \otimes_{H} d_{i j}-(-1)^{|a| b \mid} \sum_{i, j} m_{i} n_{i j(1)} \otimes m_{i j} \otimes n_{i} n_{i j(2)} \otimes_{H} e_{i j} \\
& =\sum_{i, j} s_{i} s_{i j(1)} \otimes t_{i} s_{i j(2)} \otimes t_{i j} \otimes_{H} u_{i j} .
\end{aligned}
$$

By the multiplication of CurA, we obtain

$$
\begin{aligned}
& {\left[\beta\left(f \otimes_{H^{\prime}} a\right) *\left[\left(g \otimes_{H^{\prime}} b\right) *\left(l \otimes_{H^{\prime}} c\right)\right]\right] } \\
- & (-1)^{|a| b \mid}\left((\sigma \otimes i d) \otimes_{H} i d\right)\left[\beta\left(g \otimes_{H^{\prime}} b\right) *\left[\left(f \otimes_{H^{\prime}} a\right) *\left(l \otimes_{H^{\prime}} c\right)\right]\right] \\
= & \sum_{i, j} f g_{i j} \otimes g g_{i} l_{i j(1)} \otimes \lg _{i} l_{i j_{(2)}} \otimes_{H}\left(1 \otimes_{H^{\prime}} d_{i j}\right) \\
& -(-1)^{|a| b \mid} \sum_{i, j} f m_{i} n_{i j(1)} \otimes g m_{i j} \otimes l n_{i} n_{i j(2)} \otimes_{H}\left(1 \otimes_{H^{\prime}} e_{i j}\right) \\
= & \sum_{i, j} f s_{i} s_{i i_{1}} \otimes g t_{i} s_{i j_{2}} \otimes l t_{i j} \otimes_{H}\left(1 \otimes_{H^{\prime}} u_{i j}\right) \\
= & {\left.\left[\left(f \otimes_{H^{\prime}} a\right) *\left(g \otimes_{H^{\prime}} b\right)\right] * \beta\left(l \otimes_{H^{\prime}} c\right)\right] . }
\end{aligned}
$$

Hence (CurL, $\gamma, \beta$ ) is a Hom-Lie $H$-pseudo-superalgebra. This ends the proof.

\section{Hom-Annihilation Superalgebras}

In this section we will study the annihilation superalgebras of Hom- $H$-pseudo-superalgebras. First of all we will give the definition of $H$-differential superalgebras.

Definition 4.1. An associative superalgebra $Y$ is called an associative $H$-differential superalgebra if it is a left $H$-module such that $h\left(y y^{\prime}\right)=\left(h_{(1)} y\right)\left(h_{(2)} y^{\prime}\right)$, for all $h \in H$ and homogeneous elements $y, y^{\prime} \in Y$.

Let $Y$ be an $H$-bimodule which is a commutative associative $H$-differential superalgebra. For a left $H$-module $L$, it is easy to see that $A_{Y} L=Y \otimes_{H} L$ is a left $H$-module via $h\left(y \otimes_{H} l\right)=(h y) \otimes_{H} l$, for all $h \in H$ and $y \otimes_{H} l \in A_{Y} L$.

The definition of Hom-Lie $H$-differential-superalgebras can be obtained similarly.

Proposition 4.2. Let $Y$ be a Hom-Lie $H$-differential-superalgebra and $\left(L,[*], \alpha_{L}\right)$ a Hom-Lie $H$-pseudosuperalgebra. Then $A_{Y} L$ is a Hom-Lie $H$-differential superalgebra, where the bracket and the action are given by

$$
\begin{gathered}
{\left[y \otimes_{H} l, y^{\prime} \otimes_{H} l^{\prime}\right]=\sum_{i}\left(y f_{i}\right)\left(y^{\prime} g_{i}\right) \otimes e_{i},} \\
h\left[y \otimes_{H} l, y^{\prime} \otimes_{H} l^{\prime}\right]=\left[h_{(1)}\left(y \otimes_{H} l\right), h_{(2)}\left(y^{\prime} \otimes_{H} l^{\prime}\right)\right],
\end{gathered}
$$

for all $h \in H$ and $y \otimes_{H} l, y^{\prime} \otimes_{H} l^{\prime} \in A_{Y} L$, where $\left[l * l^{\prime}\right]=\sum_{i} f_{i} \otimes g_{i} \otimes e_{i}$.

Proof. First we shall show that $A_{Y} L$ is an H-module, but this is easy to check. It remains to verify that the conditions (1) and (2) in Definition 3.5 are satisfied. For this purpose, we take $x \otimes_{H} a, y \otimes_{H} b, z \otimes_{H} c \in A_{Y} L$, and suppose

$$
\begin{gathered}
{[b * c]=\Sigma_{i} g_{i} \otimes l_{i} \otimes_{H} a_{i},\left[\alpha_{L}(a) * a_{i}\right]=\Sigma_{j} g_{i j} \otimes l_{i j} \otimes_{H} a_{i j},} \\
{[a * c]=\Sigma_{i} m_{i} \otimes n_{i} \otimes_{H} b_{i},\left[\alpha_{L}(b) * b_{i}\right]=\Sigma_{j} m_{i j} \otimes n_{i j} \otimes_{H} b_{i j},}
\end{gathered}
$$




$$
[a * b]=\Sigma_{i} f_{i} \otimes k_{i} \otimes_{H} c_{i},\left[c_{i} * \alpha_{L}(c)\right]=\Sigma_{j} f_{i j} \otimes k_{i j} \otimes_{H} c_{i j} .
$$

Since $L$ is a Hom-Lie $H$-pseudo-superalgebra, then

$[b * a]=-(-1)^{|a| b \mid}\left(\sigma \otimes_{H} i d\right)[a * b]=-(-1)^{|a| b \mid} \sum_{i} k_{i} \otimes f_{i} \otimes_{H} c_{i}$, therefore we have

$$
\begin{aligned}
{\left[y \otimes_{H} b, x \otimes_{H} a\right] } & =-(-1)^{|a| b \mid} \sum_{i}\left(y k_{i}\right)\left(x f_{i}\right) \otimes c_{i} \\
& =-(-1)^{|a| b \mid} \sum_{i}\left(x f_{i}\right)\left(y k_{i}\right) \otimes c_{i} \\
& =-(-1)^{|a| b \mid}\left[x \otimes_{H} a, y \otimes_{H} b\right],
\end{aligned}
$$

as required. Next we verify the Hom-Jacobi identity by the following calculations:

$$
\begin{aligned}
{\left[\beta\left(x \otimes_{H} a\right),\left[y \otimes_{H} b, z \otimes_{H} c\right]\right] } & =\sum_{i}\left[x \otimes_{H} \alpha(a),\left(y g_{i}\right)\left(z l_{i}\right) \otimes_{i}\right] \\
& =\sum_{i, j}\left(x g_{i j}\right)\left(\left(\left(y g_{i}\right)\left(z l_{i}\right)\right) l_{i j}\right) \otimes_{H} a_{i j} \\
& =\sum_{i, j}\left(x g_{i j}\right)\left(y g_{i} l_{i_{j(1)}}\right)\left(z l_{i} l_{i j(2)}\right) \otimes_{H} a_{i j} .
\end{aligned}
$$

Similarly, by exchanging the status of the element $x \otimes_{H} a, y \otimes_{H} b, z \otimes_{H} c \in A_{Y} L$, we have

$$
\begin{aligned}
& {\left[\beta\left(y \otimes_{H} b\right),\left[x \otimes_{H} a, z \otimes_{H} c\right]\right]=\sum_{i, j}\left(y m_{i j}\right)\left(x m_{i} n_{i j(1)}\right)\left(z n_{i} n_{i j(2)}\right) \otimes_{H} b_{i j},} \\
& {\left[\left[x \otimes_{H} a, y \otimes_{H} b\right], \beta\left(z \otimes_{H} c\right)\right]=\sum_{i, j}\left(x f_{i} f_{i j(1)}\right)\left(y k_{i} f_{i j(2)}\right)\left(z k_{i j}\right) \otimes_{H} c_{i j} .}
\end{aligned}
$$

By the Hom-Jacobi identity of $L$, we have

$$
\begin{aligned}
& {[[a * b] * \alpha(c)]-[\alpha(a) *[b * c]]+(-1)^{|a| b \mid}\left((\sigma \otimes i d) \otimes_{H} i d\right)[\alpha(b) *[a * c]]} \\
& =\sum_{i, j} f_{i} f_{i j_{(1)}} \otimes k_{i} f_{i j(2)} \otimes k_{i j} \otimes_{H} c_{i j}-g_{i j} \otimes g_{i} l_{i j(1)} \otimes l_{i} l_{i j(2)} \otimes_{H} a_{i j} \\
& \quad+(-1)^{|a| b \mid} m_{i} n_{i j(1)} \otimes m_{i j} \otimes n_{i} n_{i j(2)} \otimes_{H} b_{i j} \\
& =0 .
\end{aligned}
$$

Hence

$$
\begin{aligned}
& {\left[\left[x \otimes_{H} a, y \otimes_{H} b\right], \beta\left(z \otimes_{H} c\right)\right]-\left[\beta\left(x \otimes_{H} a\right),\left[y \otimes_{H} b, z \otimes_{H} c\right]\right]} \\
& +(-1)^{|a| b \mid}\left((\sigma \otimes i d) \otimes_{H} i d\right)\left[\beta\left(y \otimes_{H} b\right),\left[x \otimes_{H} a, z \otimes_{H} c\right]\right] \\
& =\left(x f_{i} f_{i j(1)}\right)\left(y k_{i} f_{i j(2)}\right)\left(z k_{i j}\right) \otimes_{H} c_{i j}-\left(x g_{i j}\right)\left(y g_{i} l_{i j(1)}\right)\left(z l_{i} l_{i j(2)}\right) \otimes_{H} a_{i j} \\
& \quad+(-1)^{|a| b \mid}\left(x m_{i} n_{i j(1)}\right)\left(y m_{i j}\right)\left(z n_{i} n_{i j(2)}\right) \otimes_{H} b_{i j} \\
& =0 .
\end{aligned}
$$

So $A_{Y} L$ is a Hom-Lie $H$-differential superalgebra. This completes the proof.

Remark 4.3. In particular, when $Y=X, A(L)=A_{X} L=X \otimes_{H} L$ is a Hom-Lie $H$-differential superalgebra, we call it Hom-annihilation superalgebra of the Hom-Lie $H$-pseudo-algebra $L$ and write $a_{x}=x \otimes_{H} a$ for any homogeneous elements $a \in L$ and $x \in X$.

Remark 4.4. A similar statement holds for Hom-associative $H$-pseudo-superalgebras and Hom-modules as well. For example, if $\left(M, \rho_{M}, \alpha_{M}\right)$ is a Hom- $L$-module, then $\left(A_{Y} M, \rho_{A_{Y} M}, \beta_{M}=i d_{Y} \otimes \alpha_{M}\right)$ is a Hom- $A_{Y} L-$ module with a compatible $H$-action, where

$$
\rho_{A_{Y} M}\left(\left(x \otimes_{H} a\right) \otimes\left(y \otimes_{H} m\right)\right)=\sum_{i}\left(x f_{i}\right)\left(y g_{i}\right) \otimes_{H} e_{i},
$$

if $\rho_{M}(a \otimes m)=\sum_{i} f_{i} \otimes g_{i} \otimes_{H} e_{i}$ for any homogeneous elements $x \otimes_{H} a \in A_{Y} L$ and $y \otimes_{H} m \in A_{Y} M$. 
Proposition 4.5. Let $\left(L,[*], \alpha_{L}\right)$ be a Hom-Lie $H$-pseudo-superalgebra and $Y$ a commutative associative $H$-differential superalgebra with a right action of $H$. Then $\left(Y \otimes_{H} L,[*], i d_{Y} \otimes \alpha_{L}\right)$ is a Hom-Lie $H$-pseudosuperalgebra with bracket pseudoproduct

$$
\left[\left(x \otimes_{H} a\right) *\left(y \otimes_{H} b\right)\right]=\sum_{i} f_{i_{(1)}} \otimes k_{i_{(1)}} \otimes_{H}\left(\left(x f_{i_{(2)}}\right)\left(y k_{i_{(2)}}\right) \otimes c_{i}\right),
$$

if $[a * b]=\sum_{i} f_{i} \otimes k_{i} \otimes_{H} c_{i}$ for any homogeneous elements $x \otimes_{H} a, y \otimes_{H} b \in Y \otimes_{H} L$.

Proof. According to the bracket pseudoproduct defined above, it is easy to see that $H$-bilinearity holds. To verify the Skew-commutativity and Hom-Jacobi identity, take $x \otimes_{H} a, y \otimes_{H} b, z \otimes_{H} c \in Y \otimes L$ and suppose

$$
\begin{aligned}
& {[b * c]=\Sigma_{i} g_{i} \otimes l_{i} \otimes_{H} a_{i},\left[\alpha_{L}(a) * a_{i}\right]=\Sigma_{j} g_{i j} \otimes l_{i j} \otimes_{H} a_{i j},} \\
& {[a * c]=\Sigma_{i} m_{i} \otimes n_{i} \otimes_{H} b_{i},\left[\alpha_{L}(b) * b_{i}\right]=\Sigma_{j} m_{i j} \otimes n_{i j} \otimes_{H} b_{i j},} \\
& {[a * b]=\Sigma_{i} f_{i} \otimes k_{i} \otimes_{H} c_{i},\left[c_{i} * \alpha_{L}(c)\right]=\Sigma_{j} f_{i j} \otimes k_{i j} \otimes_{H} c_{i j} .}
\end{aligned}
$$

Since $L$ is a Hom-Lie $H$-pseudo-superalgebra, $[b * a]=-(-1)^{|a| b \mid}\left(\sigma \otimes_{H} i d\right)[a * b]=-(-1)^{|a| b \mid} \sum_{i} k_{i} \otimes f_{i} \otimes_{H} c_{i}$, therefore we have

$$
\begin{aligned}
& {\left[\left(y \otimes_{H} b\right) *\left(x \otimes_{H} a\right)\right]} \\
& =-(-1)^{a|a| b \mid} \sum_{i} k_{i_{(1)}} \otimes f_{i_{(1)}} \otimes_{H}\left(\left(y f_{i_{(2)}}\right)\left(x k_{i_{(2)}}\right) \otimes c_{i}\right) \\
& =-(-1)^{|a| b \mid} \sum_{i} k_{i_{(1)}} \otimes f_{i_{(1)}} \otimes_{H}\left(\left(x k_{i_{(2)}}\right)\left(y f_{i_{(2)}}\right) \otimes c_{i}\right) \\
& =-(-1)^{|a| b \mid}\left(\left(\sigma \otimes_{H} i d\right) \otimes i d\right) \sum_{i} f_{i_{(1)}} \otimes k_{i_{(1)}} \otimes_{H}\left(\left(x f_{i_{(2)}}\right)\left(y k_{i_{(2)}}\right) \otimes c_{i}\right) \\
& =-(-1)^{|a| b \mid}\left(\left(\sigma \otimes_{H} i d\right) \otimes i d\right)\left[\left(x \otimes_{H} a\right) *\left(y \otimes_{H} b\right)\right] .
\end{aligned}
$$

That is, the skew-commutativity holds. So it is sufficient to verify the Hom-Jacobi identity. Since

$$
\left[\left(x \otimes_{H} a\right) *\left(y \otimes_{H} b\right)\right]=\sum_{i} f_{i_{(1)}} \otimes k_{i_{(1)}} \otimes_{H}\left(\left(x f_{i_{(2)}}\right)\left(y k_{i_{(2)}}\right) \otimes c_{i}\right),
$$

we have

$$
\begin{aligned}
& {\left[\left[\left(x \otimes_{H} a\right) *\left(y \otimes_{H} b\right)\right] * \beta\left(z \otimes_{H} c\right)\right]} \\
& =\sum_{i}\left[\left(f_{i_{(1)}} \otimes k_{i_{(1)}} \otimes_{H}\left(\left(x f_{i_{(2)}}\right)\left(y k_{i_{(2)}}\right) \otimes_{H} c_{i}\right)\right) *\left(z \otimes_{H} \alpha(c)\right)\right] \\
& =\sum_{i, j} f_{i_{(1)}} f_{i j(1)} \otimes k_{i_{(1)}} f_{i j(2)} \otimes k_{i j(1)} \otimes_{H}\left(x f_{i_{(2)}} f_{i j(3)}\right)\left(y k_{i_{(2)}} f_{i j(4)}\right)\left(z k_{i j(2)}\right) \otimes_{H} c_{i j} \\
& \left.=\sum_{i, j}\left(f_{i_{(1)}} \otimes k_{i_{(1)}}\right) f_{i j(1)} \otimes k_{i j(1)} \otimes_{H}\left(\left(x f_{i_{(2)}}\right)\left(y k_{i_{(2)}}\right)\right) f_{i j(2)}\right)\left(z k_{i j(2)}\right) \otimes_{H} c_{i j} .
\end{aligned}
$$

Similarly, by exchanging the status of the element $x \otimes_{H} a, y \otimes_{H} b, z \otimes_{H} c \in A_{Y} L$, we have

$$
\begin{aligned}
& {\left[\beta\left(x \otimes_{H} a\right) *\left[\left(y \otimes_{H} b\right) *\left(z \otimes_{H} c\right)\right]\right] } \\
= & \sum_{i, j} g_{i j_{(1)}} \otimes\left(g_{i_{(1)}} \otimes l_{i_{(1)}}\right) l_{i j_{(1)}} \otimes_{H}\left(x g_{i j(2)}\right)\left(\left(\left(y g_{i_{(2)}}\right)\left(z l_{i_{(2)}}\right)\right) l_{i j_{(2)}}\right) \otimes_{H} a_{i j}, \\
{[} & \left.\beta\left(y \otimes_{H} b\right) *\left[\left(x \otimes_{H} a\right) *\left(z \otimes_{H} c\right)\right]\right] \\
= & \sum_{i, j} m_{i j_{(1)}} \otimes\left(m_{i_{(1)}} \otimes n_{i_{(1)}}\right) n_{i j_{(1)}} \otimes_{H}\left(y m_{i j_{(2)}}\right)\left(\left(\left(x m_{i_{(2)}}\right)\left(z n_{i_{(2)}}\right)\right) n_{i j(2)}\right) \otimes_{H} b_{i j} .
\end{aligned}
$$

By the Hom-Jacobi identity of $L$, we have 


$$
\begin{aligned}
& {[[a * b] * \alpha(c)]-[\alpha(a) *[b * c]]+(-1)^{|a| b \mid}\left((\sigma \otimes i d) \otimes_{H} i d\right)[\alpha(b) *[a * c]]} \\
& =\sum_{i, j} f_{i} f_{i j(1)} \otimes k_{i} f_{i j(1)} \otimes k_{i j} \otimes_{H} c_{i j}-g_{i j} \otimes g_{i} l_{i j(1)} \otimes l_{i} l_{i j(2)} \otimes_{H} a_{i j} \\
& \quad+(-1)^{|a||| b \mid}\left((\sigma \otimes i d) \otimes_{H} i d\right)\left(m_{i j} \otimes m_{i} n_{i j(1)} \otimes n_{i} n_{i j(2)} \otimes_{H} b_{i j}\right) \\
& =\sum_{i, j}\left(f_{i} \otimes k_{i}\right) f_{i j} \otimes k_{i j} \otimes_{H} c_{i j}-g_{i j} \otimes\left(g_{i} \otimes l_{i}\right) l_{i j} \otimes_{H} a_{i j} \\
& \quad+(-1)^{|a||b|}\left((\sigma \otimes i d) \otimes_{H} i d\right)\left(m_{i j} \otimes\left(m_{i} \otimes n_{i}\right) n_{i j} \otimes_{H} b_{i j}\right) \\
& =0,
\end{aligned}
$$

it follows that

$$
\begin{aligned}
& {\left[\left[x \otimes_{H} a, y \otimes_{H} b\right], \beta\left(z \otimes_{H} c\right)\right]-\left[\beta\left(x \otimes_{H} a\right),\left[y \otimes_{H} b, z \otimes_{H} c\right]\right]} \\
& +(-1)^{|a| b \mid}\left((\sigma \otimes i d) \otimes_{H} i d\right)\left[\beta\left(y \otimes_{H} b\right),\left[x \otimes_{H} a, z \otimes_{H} c\right]\right]=0 .
\end{aligned}
$$

So $A_{Y} L$ is a Hom-Lie $H$-pseudo-superalgebra. This completes the proof.

\section{Hom-Lie Conformal Superalgebras}

In this section we will reformulate the definition of Hom-Lie (or Hom-associative) H-pseudo-superalgebras. The resulting notion, equivalent to that of Hom-H-pseudo-superalgebras, will be called Hom-H-conformal superalgebras.

Let us start by racalling the definitions of the Fourier transform and the $x$-brackets in [10]. For an arbitrary Hopf algebra $H$, the Fourier transform $F: H \otimes H \rightarrow H \otimes H$ is defined by $F(f \otimes g)=f S\left(g_{(1)}\right) \otimes g_{(2)}$. $F$ is an isomorphism with an inverse given by $F(f \otimes g)=f g_{(1)} \otimes g_{(2)}$. The significance of Fourier transform $F$ is the identity

$$
f \otimes g=F^{-1} F(f \otimes g)=f S\left(g_{(1)}\right) \otimes g_{(2)} .
$$

In order to reformulate the definition of a Lie (or associative) $H$-pseudo-algebra, Bakalov, D'Andrea and Kac introduced the bracket $[a, b] \in H \otimes L$ as the Fourier transform of $[a * b]$ :

$$
[a, b]=\sum_{i} F\left(f_{i} \otimes g_{i}\right)\left(1 \otimes e_{i}\right)=f_{i} S\left(g_{i_{(1)}}\right) \otimes g_{i_{(2)}} e_{i} .
$$

That is,

$$
[a, b]=\sum_{i} h_{i} \otimes c_{i}, \text { if }[a * b]=\sum_{i}\left(h_{i} \otimes 1\right) \otimes_{H} c_{i} .
$$

Then for $x \in X=H^{*}$, the $x$-bracket is defined in [3] as follows:

$$
\left[a_{x} b\right]=(\langle S(x), \cdot\rangle)[a, b]=\sum_{i}\left\langle S(x), h_{i}\right\rangle c_{i} .
$$

Let $(L,[*], \alpha)$ be a Hom-Lie $H$-pseudo-superalgebra. For any homogeneous elements $a, b, c \in L$, suppose

$$
\begin{aligned}
& {[b * c]=\sum_{i} l_{i} \otimes 1 \otimes_{H} a_{i},\left[\alpha(a) * a_{i}\right]=\sum_{j} l_{i j} \otimes 1 \otimes_{H} a_{i j},} \\
& {[a * c]=\sum_{i} g_{i} \otimes 1 \otimes_{H} b_{i},\left[\alpha(b) * b_{i}\right]=\sum_{j} g_{i j} \otimes 1 \otimes_{H} b_{i j},} \\
& {[a * b]=\sum_{i} f_{i} \otimes 1 \otimes_{H} c_{i},\left[c_{i} * \alpha(c)\right]=\sum_{j} f_{i j} \otimes 1 \otimes_{H} c_{i j} .}
\end{aligned}
$$

Then we have

$$
[\alpha(a) *[b * c]]=\sum_{i}\left[\alpha(a) *\left(l_{i} \otimes 1 \otimes_{H} a_{i}\right)\right]=\sum_{i, j} l_{i j} \otimes l_{i} \otimes 1 \otimes_{H} a_{i j}
$$




$$
[\alpha(a),[b, c]]=\sum_{i}(\sigma \otimes i d)(i d \otimes[\alpha(a), \cdot])([b, c])=\sum_{i, j} l_{i j} \otimes l_{i} \otimes a_{i j} .
$$

Similarly, we can obtain $[\alpha(b) *[a * c]],[\alpha(b),[a, c]],[[a * b] * \alpha(c)],[[a, b], \alpha(c)]$, thus

$$
\begin{aligned}
& {[\alpha(a) *[b * c]]-[[a * b] * \alpha(c)]-(-1)^{|a||b|}\left((\sigma \otimes i d) \otimes_{H} i d\right)[\alpha(b) *[a * c]]} \\
& =\sum_{i, j} l_{i j} \otimes l_{i} \otimes 1 \otimes_{H} a_{i j}-\sum_{i, j} f_{i} f_{i j_{1}} \otimes f_{i j j_{2}} \otimes 1 \otimes_{H} a_{i j}-(-1)^{|a| b \mid} \sum_{i, j} g_{i} \otimes g_{i j} \otimes 1 \otimes_{H} c_{i j}, \\
& \\
& {[\alpha(a),[b, c]]-\left(F^{-1} \otimes i d\right)[[a, b], \alpha(c)]-(-1)^{|a| b \mid}(\sigma \otimes i d)[\alpha(b),[a, c]]} \\
& =\sum_{i, j} l_{i j} \otimes l_{i} \otimes a_{i j}-\sum_{i, j}\left(f_{i} \otimes 1\right) \Delta\left(f_{i j} \otimes_{i j}-(-1)^{|a| b \mid} \sum_{i, j} g_{i} \otimes g_{i j} \otimes c_{i j}\right. \\
& =\sum_{i, j} l_{i j} \otimes l_{i} \otimes a_{i j}-\sum_{i, j} f_{i} f_{i j_{1}} \otimes f_{i j} \otimes a_{i j}-(-1)^{|a| b \mid} \sum_{i, j} g_{i} \otimes g_{i j} \otimes c_{i j} .
\end{aligned}
$$

Therefore

$$
[\alpha(a) *[b * c]]-(-1)^{|a| b \mid}\left((\sigma \otimes i d) \otimes_{H} \text { id }\right)[\alpha(b) *[a * c]]=[[a * b] * \alpha(c)]
$$

is equivalent to

$$
[\alpha(a),[b, c]]-(-1)^{|a||b|}(\sigma \otimes i d)[\alpha(b),[a, c]]=\left(F^{-1} \otimes i d\right)[[a, b], \alpha(c)] .
$$

So the definition of Hom-Lie $H$-pseudo-superalgebra can be equivalently reformulated as follows.

Definition 5.1. A Hom-Lie $H$-conformal superalgebra is a triple $(L,[],, \alpha)$ consisting of a superspace $L$ in ${ }_{H} M$, an even operation [,]: $L \otimes L \rightarrow H \otimes L$ and an even homomorphism $\alpha \in \operatorname{Hom}_{H}(L, L)$ satisfying the following axioms:

1) $H$-sesqui-linearity:

$$
[h a, b]=(h \otimes 1)[a, b],[a, h b]=\left(1 \otimes h_{(2)}\right)[a, b]\left(S\left(h_{(1)}\right) \otimes 1\right) ;
$$

2) Skew-commutativity:

$$
[b, a]=-(-1)^{|a| b \mid} \sum_{i} S\left(h_{i_{(1)}}\right) \otimes h_{i_{(2)}} c_{i}, \text { if }[a, b]=\sum_{i} h_{i} \otimes c_{i} ;
$$

3) Hom-Jacobi identity:

$$
[\alpha(a),[b, c]]-(-1)^{|a||b|}(\sigma \otimes i d)[\alpha(b),[a, c]]=\left(F^{-1} \otimes i d\right)[[a, b], \alpha(c)] ;
$$

where $a, b, c$ are homogeneous elements in $L$ and $h \in H$.

One can also reformulate Definition 4.1 in terms of $x$-brackets $\left[a_{x} b\right]$ as below.

Definition 5.2. A Hom-Lie $H$-conformal superalgebra is a triple $(L,[],, \alpha)$ consisting of a superspace $L$ in ${ }_{H} M$, an even operation $\left[{ }_{x}\right]: L \otimes L \rightarrow L$ and an even homomorphism $\alpha \in \operatorname{Hom}_{H}(L, L)$ satisfying the following axioms:

1) Locality:

$$
\operatorname{codim}\left\{x \in X \mid\left[a_{x} b\right]=0\right\}<\infty \text { for any } a, b \in L ;
$$

2) $H$-sesqui-linearity:

$$
\left[h a_{x} b\right]=\left[a_{x h} b\right],\left[a_{x} h b\right]=h_{2}\left[a_{S\left(h_{1}\right) x} b\right]
$$

3) Skew-super commutativity:

$$
\left[b_{x} a\right]=-(-1)^{|a| b \mid} \sum_{i}\left\langle x, S\left(h_{i_{1}}\right)\right\rangle S\left(h_{i_{2}}\right)\left[b_{x_{i}} a\right]
$$

4) Hom-super Jacobi identity: 


$$
\left[\alpha(a)_{x}\left[b_{y} c\right]\right]-(-1)^{|a| b \mid}\left[\alpha(b)_{y}\left[a_{x} c\right]\right]=\left[\left[a_{x_{2}} b\right]_{y x_{1}} \alpha(c)\right] .
$$

where $x_{i}$ and $y_{i}$ are dual bases of $X$ and $H, a, b, c$ are homogeneous elements in $L, h \in H$ and $x, y \in X$.

In the following we will show that there is a simple relationship between the $x$-bracket of a Hom-Lie $H$-conformal superalgebra and the commutator in its annihilation Hom-Lie $H$-pseudo-superalgebra $A(L)$ defined in Proposition 4.5. Let $h_{i}, x_{i}$ be dual linear basis of $H$ and $X$. Then we have

$$
[a, b]=\sum_{i} S\left(h_{i}\right) \otimes\left[a_{x_{i}} b\right],[a * b]=\sum_{i}\left(S\left(h_{i}\right) \otimes 1\right) \otimes_{H}\left[a_{x_{i}} b\right] .
$$

According to Proposition 4.2, we obtain

$$
\left[a_{x}, b_{y}\right]=\sum_{i}\left[a_{x_{i}} b\right]_{\left(x S\left(h_{i}\right)\right) y}=\left[a_{x_{(2)}} b\right]_{x(1) y} .
$$

In other words,

$$
\left[a_{x} b\right]_{y}=\left[a_{x_{(2)}}, b_{S(x(1)) y}\right]=\sum_{i}\left[a_{x_{i}}, b_{\left(h_{i} S(x)\right) y}\right] .
$$

Below we give one way of constructing Hom-modules over Hom-Lie $H$-pseudo-algebras, whose proofs are similar to that in [10].

Proposition 5.3. Any Hom-module $\left(M, \rho_{M}, \alpha_{M}\right)$ over a Hom-Lie $H$-pseudo-superalgebra $\left(L,[*], \alpha_{L}\right)$ has a natural structure of a Hom- $A(L)$-module, given by $\left(x \otimes_{H} a\right) \cdot m=a_{x} m$, where

$$
a_{x} m=\left\langle S(x), f_{i} S\left(g_{i_{(1)}}\right)\right\rangle g_{i_{(2)}} e_{i}, \text { if } a * m=\sum_{i}\left(f_{i} \otimes g_{i}\right) \otimes_{H} e_{i},
$$

for all homogeneous elements $a \in L, x \in X$ and $m \in M$. This action is compatible with the action of $H$, that is, $h(b m)=\left(h_{(1)} b\right)\left(h_{(2)} m\right)$ for all homogeneous elements $b \in A(L), m \in M$ and $h \in H$, and satisfies the locality condition: $\operatorname{codim}\left\{x \in X \mid\left[a_{x} M\right]=0\right\}<\infty$ for any homogeneous elements $a \in L$ and $m \in M$.

Conversely, any $\operatorname{Hom}-A(L)$-module $\left(M, \rho_{M}, \alpha_{M}\right)$ satisfying the above conditions has a natural structure of an Hom- $L$-module, given by

$$
a * m=\sum_{i}\left(S\left(h_{i}\right) \otimes 1\right) \otimes_{H} a_{x_{i}} m,
$$

where $h_{i}$ and $x_{i}$ are dual linear basis of $H$ and $X$.

\section{Cohomology of Hom-Lie H-Pseudo-Superalgebras}

In this section, we will consider the cohomology of Hom-Lie $H$-pseudo-superalgebras, generalizing the results of Hom-Lie $H$-pseudoalgebras and Lie superalgebras.

Let $\left(L,[],, \alpha_{L}\right)$ be a Hom-Lie $H$-pseudo-superalgebra, $\left(M, \rho_{M}, \alpha_{M}\right)$ is a Hom- $L$-module. Let $n \geq 1$ be a natural number and let $C_{\text {Hom }}^{n}(L, M)$ be the superspace of all homogeneous skew-symmetric cochains $\gamma \in \operatorname{Hom}_{H^{\otimes n}}\left(L^{\otimes n}, H^{\otimes n} \otimes_{H} M\right)$ satisfies

$$
\begin{aligned}
& \left(i d_{H}^{n} \otimes_{H} \alpha_{M}\right) \gamma\left(a_{1} \otimes a_{2} \otimes \cdots \otimes a_{n-1} \otimes a_{n}\right) \\
& =\gamma\left(\alpha_{L}\left(a_{1}\right) \otimes \alpha_{L}\left(a_{2}\right) \otimes \cdots \otimes \alpha_{L}\left(a_{n-1}\right) \otimes \alpha_{M}\left(a_{n}\right)\right) .
\end{aligned}
$$

Explicitly, $\gamma$ has the following defining properties:

1) $H$-polylinearity: For any $h \in H$ and $a_{i} \in L$,

$$
\begin{aligned}
& \gamma\left(h_{1} a_{1} \otimes h_{2} a_{2} \otimes \cdots \otimes h_{n-1} a_{n-1} \otimes h_{n} a_{n}\right) \\
& =\left(\left(h_{1} \otimes h_{2} \otimes \cdots \otimes h_{n-1} \otimes h_{n}\right) \otimes_{H} \otimes 1\right) \gamma\left(a_{1} \otimes a_{2} \otimes \cdots \otimes a_{n-1} \otimes a_{n}\right) .
\end{aligned}
$$

2) Skew-supersymmetry: For any $a_{i} \in L$, 


$$
\begin{aligned}
& \gamma\left(a_{1} \otimes \cdots \otimes a_{i+1} \otimes a_{i} \otimes \cdots \otimes a_{n}\right) \\
& =-(-1)^{\left|a_{i}\right|\left|a_{i+1}\right|}\left(\sigma_{i, i+1} \otimes_{H} i d\right) \gamma\left(a_{1} \otimes \cdots \otimes a_{i} \otimes a_{i+1} \otimes \cdots \otimes a_{n}\right),
\end{aligned}
$$

where $\sigma_{i, i+1}: H^{\otimes n} \rightarrow H^{\otimes n}$ is the transposition of the $i$ th and $(i+1)$ st factors.

The map $\gamma$ is called even (resp. odd) when $\gamma\left(a_{1} \otimes a_{2} \otimes \cdots \otimes a_{n}\right) \in M_{0} \quad\left(\right.$ resp. $\left.\gamma\left(a_{1} \otimes a_{2} \otimes \cdots \otimes a_{n}\right) \in M_{1}\right)$ for all even (resp. odd) elements $a_{1} \otimes a_{2} \otimes \cdots \otimes a_{n} \in H^{\otimes n}$, where the parity of the element $a_{1} \otimes a_{2} \otimes \cdots \otimes a_{n}$ is $\left|a_{1}\right|+\left|a_{2}\right|+\cdots+\left|a_{n}\right|$. We denote the parity of the map $\gamma$ by $|\gamma|$.

For $n \geq 1$, the map $d: C_{\text {Hom }}^{n}(L, M) \rightarrow C_{\text {Hom }}^{n+1}(L, M)$ is defined as follows:

$$
\begin{aligned}
& (d \gamma)\left(a_{1} \otimes \cdots \otimes a_{i+1} \otimes a_{i} \otimes \cdots \otimes a_{n} \otimes a_{n+1}\right) \\
& =\sum_{1 \leq i \leq n+1}(-1)^{i+1}(-1)^{\left|a_{i}\right|\left(\gamma|+| a_{1}|+\cdots+| a_{i-1} \mid\right)}\left(\sigma_{1 \rightarrow i} \otimes_{H} i d\right) \alpha_{L}^{n-1}\left(a_{i}\right) * \gamma\left(a_{1} \otimes \cdots \otimes \hat{a}_{i} \otimes \cdots \otimes a_{n+1}\right) \\
& \quad+\sum_{1 \leq i<j \leq n+1}(-1)^{i+j}(-1)^{\left|a_{i}\right|\left(\left|a_{1}\right|+\cdots+\left|a_{i-1}\right|\right)}(-1)^{\left|a_{j}\right|\left(\left|a_{1}\right|+\cdots+\left|a_{i-1}\right|+\left|a_{i+1}\right|+\cdots+\left|a_{j-1}\right|\right)}\left(\sigma_{1 \rightarrow i, 2 \rightarrow j} \otimes_{H} i d\right) \\
& \quad \times \gamma\left(\left[a_{i} * a_{j}\right] \otimes \alpha_{L}\left(a_{1}\right) \otimes \cdots \otimes \hat{a}_{i} \otimes \cdots \otimes \hat{a}_{j} \otimes \cdots \otimes \alpha\left(a_{n+1}\right)\right),
\end{aligned}
$$

where $\sigma_{1 \rightarrow i}$ is the permutation $h_{i} \otimes h_{1} \otimes \cdots \otimes h_{i-1} \otimes h_{i+1} \otimes \cdots \otimes h_{n+1} \mapsto h_{1} \otimes \cdots \otimes h_{n+1}, \quad \sigma_{1 \rightarrow i, 2 \rightarrow j}$ is the permutation $h_{i} \otimes h_{j} \otimes h_{1} \otimes \cdots \otimes h_{i-1} \otimes h_{i+1} \otimes \cdots \otimes h_{j-1} \otimes h_{j+1} \otimes \cdots \otimes h_{n+1} \mapsto h_{1} \otimes \cdots \otimes h_{n+1}$, and the sign $\wedge$ indicates that the element below it must be omitted. In particular, for $n=1$ we have

$$
(d \gamma)\left(a_{1}, a_{2}\right)=-\gamma\left(\left[a_{1} * a_{2}\right]\right)+(-1)^{|\gamma| a_{1} \mid} a_{1} * \gamma\left(a_{2}\right)-(-1)^{\left|a_{2}\right|\left(|\gamma|+\left|a_{1}\right|\right)}\left(\sigma_{1 \rightarrow 2} \otimes_{H} \text { id }\right) \alpha_{2} * \gamma\left(a_{1}\right),
$$

and for $n=2$ we obtain

$$
\begin{aligned}
& (d \gamma)\left(a_{1} \otimes a_{2} \otimes a_{3}\right)=(-1)^{|\gamma| a_{1} \mid} \alpha_{L}\left(a_{1}\right) * \gamma\left(a_{2} \otimes a_{3}\right) \\
& -(-1)^{\left|a_{2}\right|\left(|\gamma|+\left|a_{1}\right|\right)}\left(\sigma_{1 \rightarrow 2} \otimes_{H} \text { id }\right) \alpha_{L}\left(a_{2}\right) * \gamma\left(a_{1} \otimes a_{3}\right) \\
& +(-1)^{\left|a_{3}\right|\left(|\gamma|+\left|a_{1}\right|+\left|a_{2}\right|\right)}\left(\sigma_{1 \rightarrow 3} \otimes_{H} \text { id }\right) \alpha_{L}\left(a_{3}\right) * \gamma\left(a_{1} \otimes a_{2}\right) \\
& -(-1)^{\left|a_{3}\right| a_{2} \mid}\left(\sigma_{1 \rightarrow 2,2 \rightarrow 3} \otimes_{H} \text { id }\right) \gamma\left(\left[a_{2} * a_{3}\right] \otimes \alpha_{L}\left(a_{1}\right)\right) \\
& +(-1)^{\left|a_{3}\right| a_{1} \mid}\left(\sigma_{1 \rightarrow 1,2 \rightarrow 3} \otimes_{H} \text { id }\right) \gamma\left(\left[a_{1} * a_{3}\right] \otimes \alpha_{L}\left(a_{2}\right)\right)-\gamma\left(\left[a_{1} * a_{2}\right] \otimes \alpha_{L}\left(a_{3}\right)\right) .
\end{aligned}
$$

The fact that $d^{2}=0$ is most easily checked and the same argument is in the usual Lie superalgebra case in [26] [36] [37] and Hom-Lie $H$-pseudoalgebra case in [34]. The cohomology of the resulting complex $C_{\text {Hom }}^{*}(L, M)$ is called the cohomology of $\left(L,[*], \alpha_{L}\right)$ with coefficients in $\left(M, \rho_{M}, \alpha_{M}\right)$ and is denoted by $H_{\text {Hom }}^{\circ}(L, M)$.

One can also modify the above definition by replacing everywhere $\otimes_{H}$ by $\otimes$. Let $\tilde{C}_{\text {Hom }}^{n}(L, M)$ consist of all skew-symmetric cochains $\gamma \in \operatorname{Hom}_{H^{\otimes n}}\left(L^{\otimes n}, H^{\otimes n} \otimes M\right)$. Then we can define a differential

$\tilde{d}: \tilde{C}_{\text {Hom }}^{n}(L, M) \rightarrow \tilde{C}_{\text {Hom }}^{n+1}(L, M)$ by (6.1) with $\otimes_{H}$ replaced by $\otimes$ everywhere; then again $\tilde{d}^{2}=0$. The corresponding cohomology $\tilde{H}_{\text {Hom }}^{\cdot}(L, M)$ will be called the basic cohomology of $\left(L,[*], \alpha_{L}\right)$ with coefficients in $\left(M, \rho_{M}, \alpha_{M}\right)$. In contrast, $H_{\text {Hom }}^{\bullet}(L, M)$ is sometimes called the reduced cohomology.

In the following we will show that the cohomology theory of Hom-Lie $H$-pseudo-superalgebras describes extensions and deformations, just as any cohomology theory.

Theorem 6.1. Let $\left(L,[*], \alpha_{L}\right)$ be a multiplicative Hom-Lie $H$-pseudo-superalgebra, and $\left(M, \rho_{M}, \alpha_{M}\right)$ be a Hom- $L$-module, considering a Hom-Lie $H$-pseudo-superalgebra with respect to the zero pseudobracket, then the equivalence classes of $H$-split abelian extensions

$$
0 \rightarrow M \rightarrow \hat{L} \rightarrow L \rightarrow 0
$$

of the Hom-Lie $H$-pseudo-superalgebra $\left(L,[*], \alpha_{L}\right)$ correspond bijectively to $H_{H o m}^{2}(L, M)_{0}$, the homogeneous component of degree zero of the reduced cohomology $H_{\text {Hom }}^{2}(L, M)$.

Proof. Let $0 \rightarrow M \rightarrow \hat{L} \rightarrow L \rightarrow 0$ be an extension of $L$-modules, which is split over $H$. Choosing a splitting $\hat{L}=L \oplus M=\{l+m \mid l \in L, m \in M\}$ as an $H$-module, and denoting the pseudobracket of $\hat{L}$ by $[a \hat{*} b]$, we have for all $a, b \in L, m, n \in M$ : 


$$
[a \hat{*} m]=[a * m],[m \hat{*} n]=0,[a \hat{*} b]-[a * b]=: \gamma(a \otimes b) \in H^{\otimes 2} \otimes_{H} M .
$$

It is not hard to verify that $\gamma$ is a homogeneous 2-cochain of degree zero, i.e., $\gamma \in C_{\text {Hom }}^{2}(L, M)_{0}$. The Homsuper Jacobi identity of $L$ and $\hat{L}$ implies $d \gamma=0$ in the sense of (6.1).

Conversely, given an element of $H_{H \text { Hom }}^{2}(L, M)_{0}$, we can choose a representative $\gamma \in C_{\text {Hom }}^{2}(L, M)_{0}$ and define an action $[\hat{*}]$ by (6.2). Then $[\hat{*}]$ depends only on the $\gamma$.

\section{Acknowledgements}

The paper is partially supported by the Project Funded by China Postdoctoral Science Foundation (No. 2015M571725), the Key University Science Research Project of Anhui Province (Nos. KJ2015A294 and KJ2014A183) and the NSF of Chuzhou University (Nos. 2015qd01, 2014qd008 and 2014PY08).

\section{References}

[1] Bakalov, B., Kac, V.G. and Voronov, A.A. (1999) Cohomology of Conformal Algebras. Communications in Mathematical Physics, 200, 561-598. http://dx.doi.org/10.1007/s002200050541

[2] D’Andrea, A. and Kac, V.G. (1998) Structure Theory of Finite Conformal Algebras. Selecta Mathematica, 4, 377-418.

[3] Kac, V.G. (1977) Lie Superalgebras. Advances in Mathematics, 26, 8-96. http://dx.doi.org/10.1016/0001-8708(77)90017-2

[4] Kac, V.G. (1998) Vertex Algebras for Beginners. 2nd Edition, American Mathematical Society, Providence. http://dx.doi.org/10.1090/ulect/010

[5] Kac, V.G. (1997) The Idea of Locality. In: Doebner, H.-D., et al., Eds., Physical Applications and Mathematical Aspects of Geometry, Groups and Algebras, World Scientific, Singapore, 16-32.

[6] Kac, V.G. Formal Distribution Algebras and Conformal Algebras. XII-th International Congress in Mathematical Physics (ICMP’97), Brisbane, 8 April 1999, 80-97.

[7] Yuan, L.M. (2014) Hom Gel'fand-Dorfman Bialgebras and Hom-Lie Conformal Algebra. Journal of Mathematical Physics, 55, Article ID: 043507. http://dx.doi.org/10.1063/1.4870870

[8] Yuan, L.M. (2016) A Lie Conformal Algebra of Block Type. arXiv, No. 1601.07388.

[9] Borcherds, R.E. (1986) Vertex Algebras, Kac-Moody Algebras, and the Monster. Proceedings of the National Academy of Sciences of the United States of America, 83, 3068-3071. http://dx.doi.org/10.1073/pnas.83.10.3068

[10] Bakalov, B., D’Andrea, A. and Kac, V.G. (2001) Theory of Finite Pseudoalgebras. Advances in Mathematics, 162, 1-140. http://dx.doi.org/10.1006/aima.2001.1993

[11] Bakalov, B., D’Andrea, A. and Kac, V.G. (2006) Irreducible Modules over Finite Simple Lie Pseudoalgebras. I. Primitive Pseudoalgebras of Type $W$ and S. Advances in Mathematics, 204, 278-346. http://dx.doi.org/10.1016/j.aim.2005.07.003

[12] Bakalov, B., D'Andrea, A. and Kac, V.G. (2013) Irreducible Modules over Finite Simple Lie Pseudoalgebras II. Primitive Pseudoalgebras of Type K. Advances in Mathematics, 232, 188-237. http://dx.doi.org/10.1016/j.aim.2012.09.012

[13] Boyallian, C. and Liberati, J.I. (2012) On Pseudo-Bialgebras. Journal of Algebra, 372, 1-34. http://dx.doi.org/10.1016/j.jalgebra.2012.08.009

[14] Sun, Q.X. (2012) Generalization of H-Pseudoalgebraic Structures. Journal of Mathematical Physics, 53, Article ID: 012105. http://dx.doi.org/10.1063/1.3665708

[15] Hartwig, J., Larsson, D. and Silvestrov, S. (2006) Deformations of Lie Algebras Using $\sigma$-Derivations. Journal of Algebra, 295, 314-361. http://dx.doi.org/10.1016/j.jalgebra.2005.07.036

[16] Larsson, D. and Silvestrov, S. (2007) Quasi-Hom-Lie Algebras, Central Extensions and 2-Cocycle-Like Identities. Journal of Algebra, 288, 321-344. http://dx.doi.org/10.1016/j.jalgebra.2005.02.032

[17] Yau, D. (2009) Hom-Algebras and Homology. Journal of Lie Theory, 19, 409-421.

[18] Yau, D. (2008) Enveloping Algebras of Hom-Lie Algebras. Journal of Generalized Lie Theory and Applications, 2, 95-108.

[19] Yau, D. (2010) Hom-Bialgebras and Comodule Algebras. International Electronic Journal of Algebra, 8, 45-64.

[20] Cheng, Y.S. and Yang, H.Y. (2010) Low-Dimensional Cohomology of q-deformed Heisenberg-Virasoro Algebra of Hom-Type. Frontiers of Mathematics in China, 5, 607-622. http://dx.doi.org/10.1007/s11464-010-0063-z

[21] Sheng, Y.H. (2012) Representations of Hom-Lie Algebras. Algebras and Representation Theory, 15, 1081-1098. 
http://dx.doi.org/10.1007/s10468-011-9280-8

[22] Sheng, Y.H. and Bai, C.M. (2014) A New Approach to Hom-Lie Bialgebras. Journal of Algebra, 399, 232-250. http://dx.doi.org/10.1016/j.jalgebra.2013.08.046

[23] Yuan, L.M. (2012) Hom-Lie Color Algebra Structures. Communications in Algebra, 40, 575-592. http://dx.doi.org/10.1080/00927872.2010.533726

[24] Makhlouf, A. and Silvestrov, S. (2008) Hom-Algebra Structures. Journal of Generalized Lie Theory and Applications, 3, 51-64. http://dx.doi.org/10.4303/jglta/S070206

[25] Makhlouf, A. and Silvestrov, S. (2009) Hom-Lie Admissible Hom-Coalgebras and Hom-Hopf Algebras. In: Silvestrov, S., Paal, E., Abramov, V. and Stolin, A., Eds., Generalized Lie Theory in Mathematics, Physics and Beyond, Springer-Verlag, Berlin, 189-206.

[26] Makhlouf, A. and Silvestrov, S. (2010) Hom-Algebras and Hom-Coalgebras. Journal of Algebra and Its Applications, 9, 553-589. http://dx.doi.org/10.1142/S0219498810004117

[27] Caenepeel, S. and Goyvaerts, I. (2012) Monoidal Hom-Hopf Algebras. Communications in Algebra, 40, 1933-1950.

[28] Chen, Y.Y., Wang, Z.W. and Zhang, L.Y. (2013) The FRT-Type Theorem for the Hom-Long Equation. Communications in Algebra, 41, 3931-3948. http://dx.doi.org/10.1080/00927872.2013.781614

[29] Chen, Y.Y., Wang, Z.W. and Zhang, L.Y. (2014) Quasitriangular Hom-Hopf Algebras. Colloquium Mathematicum, 137, 67-88. http://dx.doi.org/10.4064/cm137-1-5

[30] Gohr, A. (2010) On Hom-Algebras with Surjective Twisting. Journal of Algebra, 324, 1483-1491. http://dx.doi.org/10.1016/j.jalgebra.2010.05.003

[31] You, M.M. and Wang, S.H. (2014) Constructing New Braided T-Categories over Monoidal Hom-Hopf Algebras. Journal of Mathematical Physics, 55, 111701. http://dx.doi.org/10.1063/1.4900824

[32] Zhang, X.H. and Wang, S.H. (2015) Weak Hom-Hopf Algebras and Their (Co)Representations. Journal of Geometry and Physics, 94, 50-71. http://dx.doi.org/10.1016/j.geomphys.2014.11.014

[33] Ammar, F. and Makhlouf, A. (2010) Hom-Lie Superalgebras and Hom-Lie Admissible Superalgebras. Journal of Algebra, 324, 1513-1528. http://dx.doi.org/10.1016/j.jalgebra.2010.06.014

[34] Scheunert, M. and Zhang, R.B. (1998) Cohomology of Lie Superalgebras and Their Generalizations. Journal of Mathematical Physics, 39, 5024-5061. http://dx.doi.org/10.1063/1.532508

[35] Sweedler, M.E. (1969) Hopf Algebras. Mathematics Lecture Notes Series. Benjamin, New York.

[36] Bahturin, Y., Mikhalev, D., Zaicev, M. and Petrogradsky, V. (1992) Infinite Dimensional Lie Superalgebras. Walter de Gruyter Publisher, Berlin. http://dx.doi.org/10.1515/9783110851205

[37] Kassel, C. (1995) Quantum Groups. Graduate Texts in Mathematics. Springer-Verlag, New York. http://dx.doi.org/10.1007/978-1-4612-0783-2 